\title{
Peer Learning, Research, and Support in Times of the COVID-19 Pandemic: a Case Study of the Early Career Psychiatrists Model
}

\author{
Ramdas S. Ransing ${ }^{1}$ (D) Mariana Pinto da Costa $^{2} \cdot$ Victor Pereira-Sanchez $^{3} \cdot$ Frances Adiukwu $^{4} \cdot$ Laura Orsolini $^{5}$. \\ Jairo M. Gonzalez-Diaz ${ }^{6}$. Amine Larnaout ${ }^{7}$ • Paolo Grandinetti ${ }^{8}$ - Drita Gashi Bytyçi ${ }^{9}$ • Joan Soler-Vidal ${ }^{10}$. \\ Zulvia Syarif $^{11}$. Ganesh Kudva Kundadak ${ }^{12} \cdot$ Mohammadreza Shalbafan $^{13} \cdot$ Marwa Nofal $^{14} \cdot$ Rodrigo Ramalho $^{15}$
}

Received: 15 November 2020 / Accepted: 20 April 2021 / Published online: 12 May 2021

(C) Academic Psychiatry 2021

In times of global crisis such as the COVID-19 pandemic, international collaboration is key as it enables clinicians, academics, and researchers to access current knowledge, expertise, and skills; gain new perspectives; and build relationships with other colleagues [1]. However, earlycareer psychiatrists (ECPs) around the world have access to limited opportunities to contribute to global mental health and research through international collaboration, particularly in low- and middle-income countries (LMICs). Lack of trained human resources and insufficient training opportunities, coupled with underdeveloped mental health research capacity, limited academic infrastructures, and financial constraints, are key barriers [2].

Importantly, many national and international organizations, such as the World Health Organization (WHO) and the World Psychiatric Association (WPA), have created several initiatives aimed at strengthening international research collaboration among ECPs [3, 4]. Nevertheless, these initiatives are commonly centered on a model of mentor-mentee or senior-junior, in which an experienced professional supports a less experienced one. Although

Ramdas S. Ransing

ramdas_ransing123@yahoo.co.in

BKL Walawalkar Rural Medical College, Ratnagiri, Maharashtra, India

2 Queen Mary University of London, London, UK

3 NYU Grossman School of Medicine, New York, USA

4 University of Port Harcourt Teaching Hospital, Port Harcourt, Nigeria

5 Polytechnic University of Marche, Ancona, Italy

6 Universidad del Rosario Clínica Nuestra Señora de la Paz, Bogota, Colombia these models can be productive and successful, they may also have limitations when they cannot provide adequate mentorship and might introduce a hierarchical gap, leading to potential inhibition of rapport among mentor-mentee and may cause work stress [5]. Other barriers are the deficiency of mentors in LMICs, restrictions on the number of mentees per mentor from training institutes, and overburdened mentors $[5,6]$.

Alternative models, such as peer-led learning, could overcome some of these barriers. In fact, a peer-led learning model offers unique opportunities that emphasize empathy and a sense of equity and expertise [7]. They often have more acceptability and usefulness at early career stages than traditional mentoring relationships and lead to longevity in terms of relationships [7]. Furthermore, remote or online peer networks can be quickly established when physical distance is an obstacle [8]. However, there is scarce literature on peer-led learning models. To fill this gap, this article describes a successful ECPs peer-led learning model to conduct research leading to scientific publications.

7 Tunis El Manar University, Tunis, Tunisia

8 ASL Teramo, Teramo, Italy

9 Hospital and University Clinical Service of Kosovo, Prizren, Kosovo

10 Fidmag Research Foundation, Barcelona, Spain

11 Tarakan General Hospital, Jakarta, Indonesia

12 Institute of Mental Health, Singapore, Singapore

13 Iran University of Medical Sciences, Tehran, Iran

14 Helwan Mental Health Hospital, Helwan, Cairo, Egypt

15 University of Auckland, Auckland, New Zealand 


\section{The Birth of a Collaborative Group}

Due to the COVID-19 pandemic, the world faced increased mental health needs that public health and healthcare systems were not prepared to address promptly [9]. ECPs were facing many challenges, including inadequate training in telepsychiatry, the non-availability of relevant personal protective equipment (PPE) to assess patients with COVID-19specific symptoms, redeployment to COVID-19 medical care services, fear of COVID-19 infection, struggles around work-life balance, and a lack of time to keep up with a rapidly growing body of research and clinical guidelines $[10$, $11]$.

At the early stages of the pandemic, the first author (RSR) extended an informal invitation to ECPs who were interested in joining a WhatsApp ${ }^{\circledR}$ group to share their experiences and collaborate. The objective of the group was to support ECPs in sharing new knowledge and preparing brief scientific documents to be shared among fellow ECPs to improve their practices during the pandemic. The invitation was posted in four WhatsApp ${ }^{\circledR}$ groups, which had been created during previous WPA conferences and leadership courses and were maintained by ECPs to keep in touch and share scientific knowledge. There were no stringent inclusion or exclusion criteria. However, attention was paid to recruit a minimum of one person from each WHO region, and at a country level, to recruit only one person per country, except for Italy (since this country was severely affected by COVID-19 at the time when the group was formed and Italian ECPs were particularly overburdened due to clinical work and redeployment). Group participants were accepted in the group on a "first come, first served" basis.

As a result, a closed WhatsApp ${ }^{\circledR}$ group of 17 ECPs (Table 1) was formed. Members' representation was diverse in terms of age (30-40 years), gender (male $=11$, female $=6$ ), professional experience (1-7 years), and countries of origin covering all WHO regions and economic strata (Table 1). Group members were psychiatric trainees, doctoral fellows, post-doctoral fellows, clinicians, and academics. When joining the WhatsApp ${ }^{\circledR}$ group, all members were provided with some instructions (e.g., the purpose of the group and general rules, such as not to spam, respect privacy, and maintain confidentiality) and were asked to share some information about themselves and their countries.

\section{The Research Cycle}

Once in the group, all members were encouraged to share recently published articles, relevant links or websites, videos, or challenges they were experiencing during the pandemic, or ideas about how to overcome these challenges. These communications were streamlined to identify a relevant topic, but on a few occasions, the discussion was guided by the first author. Whenever a topic was identified as relevant from a clinical or service perspective, it was then considered suitable for further exploration. The process of selecting a topic was dependent on the person leading that particular topic (a member of the group who proposed the topic and introduced its relevance) and the group's recognition of the topic as relevant.

Once a topic was identified, a group member was assigned the role of lead and corresponding author of the potential publication. This person was the one who originally pitched the idea or someone with a combination of an invested interest in the topic and time availability to prepare an initial draft. The lead then invited one or more co-leads, i.e., team members who would collect responses from others, pool knowledge, and review the relevant literature. This information was shared in a shared Google $₫$ drive folder. The lead and co-lead(s) prepared a first draft of the manuscript based on the countryspecific input from each collaborator and then shared it with other group members who expressed interest in that topic. In that way, all collaborators contributed both with content and with reviewing the manuscript.

The International Committee of Medical Journal and Editors' (ICMJE) criteria were used to assess authorship and sequence eligibility. Leads and co-leads were responsible for evaluating each team member's contribution, the authorship sequence, and the use of a suitable credit system $[12,13]$. In case of discrepancy, the lead author of the particular topic made the final decision, as per the ICMJE criteria [12]. In case of revision or rejection from the journal, the manuscript was revised with inputs from contributing authors.

There are various examples of this process (Table 2). For instance, a query on how to measure COVID-19-related mental health issues in clinical practice led to a discussion on the "pros" and "cons" of the different instruments available at that time and an eventual publication in the form of a letter to the editor by one of the authors (RSR) [11]. Similarly, a discussion on how to assimilate the relevant scales in one's clinical practice led to another publication on a "toolkit," led by another author (FA). Every stage of the process was carried out in an expedited manner and within a restricted time frame due to the pandemic, responding to the need for rapid and good quality scientific literature helpful to ECPs across the world.

Published articles were then disseminated through social media platforms. The author MPC disseminated all publications through the social media accounts of the WPA-ECPs Section (Facebook ${ }^{\circledR}$ and Twitter ${ }^{\circledR}$ ). The author VPS prepared and initiated the unique series of brief videos of about 5 min or less (Our Science in a Nutshell series), in which authors presented the published papers. In addition, several co-authors presented the group's findings in various conferences and meetings (Table 1). 
Table 1 ECP's peer learning model

\begin{tabular}{|c|c|}
\hline Project name & COVID-19 and mental health \\
\hline Duration & 6 months (March 20, 2020, to Sept 19, 2020) \\
\hline Medium of communication & WhatsApp ${ }^{\circledR}$ (most commonly), Email, Google docs, Google ${ }^{\circledR}$ forms, Google $®$ Drive, Zoom $®$ (rarely) \\
\hline Participants' countries & $\begin{array}{l}\text { Italy (2), India, UK/Portugal, USA, Tunisia, Singapore, Lebanon, India, Egypt, Iran, Kosovo, } \\
\text { New Zealand, Brazil, Colombia, Spain, Indonesia }\end{array}$ \\
\hline Outcome & 11 publications, 12 presentations \\
\hline Dissemination of findings & 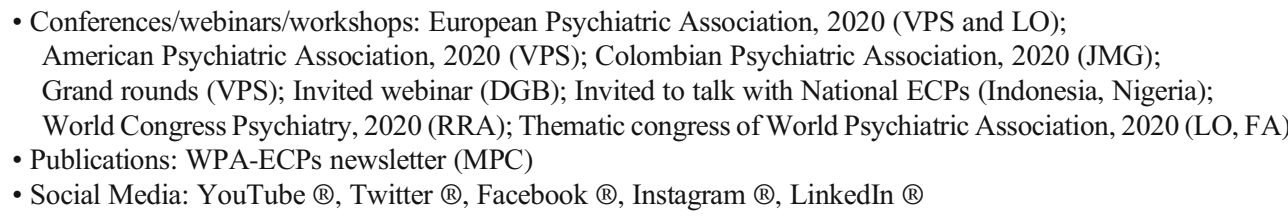 \\
\hline ECP's new initiatives with outcomes & $\begin{array}{l}\text { - Mental Health Research Network of Egypt [MN]: training } \\
\text { - COVID-19 and Mental Health-India [RSR]: } 3 \text { publications } \\
\text { - WPA-Preventive Psychiatry and Mental Health Promotion Section [RRA] \{planned\} } \\
\text { - Research network for original research: Albania, Nigeria, India, Italy, and Iran [RSR] } \\
\text { - COVID-19 think tank-Global [VPS]: } 3 \text { publications , } 4 \text { under-review } \\
\text { - Experience sharing: Iran and Colombia [MS and JMG]: } 3 \text { publications }\end{array}$ \\
\hline
\end{tabular}

\section{The Outcome}

The primary objective of this project was to deepen the knowledge about COVID-19-related mental health issues and meaningfully contribute to the field of mental health during a pandemic. The primary visible outcome was impactful research measured as publications in international peer-reviewed journals, with citations, the number of presentations in conferences or webinars, and the research interest (measured by ResearchGate $($ ) of each article (Table 2). Out of these outcomes, citations, and research interests were considered external quality indices. Impressively, 11 publications in peerreviewed and indexed journals (Table 2) and 12 presentations in scientific meetings (Table 1) have arisen through this model in six months. MN and VPS organized social gatherings using Zoom®, which also provided emotional peer support and fostered camaraderie. The pilot project was concluded on September 20, 2020, after six months. Still, the network has inspired many team members and ECPs to initiate research activities or educational collaboration, growing outside of the group, which can also be considered as an index of its success (Table 1).

\section{Discussion}

This international and web-based collaboration has been invaluable in supporting ECPs across the world, helping to further the body of scientific work on mental health aspects of the COVID-19 pandemic and improving the research and publication competence among ECPs. The model's success was grounded on many factors such as individual motivation, trust among team members, openness to collaborative work, transparency, mutual respect, active involvement of team members in contribution and learning, a helping attitude towards each other, efficient leads and co-leads for each topic, and effective communication. All group efforts aimed to ensure the clinical utility of our publications across all WHO regions and for a variety of clinical and sociocultural contexts (Table 2). The whole process helped team members acknowledge, understand, and critically discuss the global and context-specific aspects of prevention, assessment, and intervention in mental health during a pandemic.

Despite variations in training, culture, languages, and experiences, we treated one another as equals. Some of our colleagues had limited experience with conducting literature searches and reviews, manuscript writing and preparation, research collaboration, journal selection (e.g., being mindful of predatory journals), and publication ethics (e.g., evaluation of the contribution, authorship management) before joining the network. This model has provided an opportunity to learn from peers on these domains and shape an international collaborative network for future global mental health research. Some authors attempted their first few publications with this group, and the process of writing a scientific article that may be published has encouraged them to contribute to this process. After 6 months, all team members reported increased confidence in international collaboration and dealing with research in emergency or epidemic situations in their countries. Moreover, a very active and collaborative exchange of information improved all members' knowledge about available online resources and fellowships and other academic and professional opportunities.

During the pandemic, we acknowledged the need not only for worldwide collaboration to understand the complexities of the outbreak but also to disseminate the information at 
Table 2 Overview of publications under ECP's peer learning model

\begin{tabular}{|c|c|c|c|c|c|c|c|}
\hline $\begin{array}{l}\text { Sr } \\
\text { no }\end{array}$ & Published article & $\begin{array}{l}\text { Salient features of the } \\
\text { publication }\end{array}$ & Credit system & $\begin{array}{l}\text { Research } \\
\text { interest }\end{array}$ & $\begin{array}{l}\text { Journal } \\
\text { (type/region/country) }\end{array}$ & $\begin{array}{l}\text { Participant } \\
\text { from } \\
\text { number of } \\
\text { countries }\end{array}$ & $\begin{array}{l}\text { No of } \\
\text { citations } \\
\text { (Google } \\
\text { scholar)\# }\end{array}$ \\
\hline 1 & $\begin{array}{l}\text { A conceptual framework for } \\
\text { mental health } \\
\text { interventions during the } \\
\text { COVID-19 pandemic }\end{array}$ & $\begin{array}{l}\text { Framework, conceptualization } \\
\text { of mental } \\
\text { health services during } \\
\text { COVID-19 pandemic }\end{array}$ & SDC+FLAE & 28.7 & Asian & 16 & 73 \\
\hline 2 & $\begin{array}{l}\text { COVID-19: patients and } \\
\text { workforce }\end{array}$ & $\begin{array}{l}\text { Perspective, analysis of } \\
\text { existing infrastructure }\end{array}$ & $\mathrm{EC}$ & 27.2 & International & 16 & 52 \\
\hline 3 & $\begin{array}{l}\text { Measuring COVID-19 related } \\
\text { mental health issues }\end{array}$ & $\begin{array}{l}\text { Review of newly developed } \\
\text { scales }\end{array}$ & SDC+FLAE & 32.8 & International & 17 & 31 \\
\hline 4 & $\begin{array}{l}\text { Telepsychiatry and healthcare } \\
\text { access inequities }\end{array}$ & $\begin{array}{l}\text { Viewpoint, global } \\
\text { perspective, } \\
\text { psychiatric services }\end{array}$ & $\mathrm{EC}$ & 13.1 & Asian & 16 & 18 \\
\hline 5 & $\begin{array}{l}\text { Yoga for COVID-19 related } \\
\text { mental health issues }\end{array}$ & $\begin{array}{l}\text { Review, preventive, } \\
\text { promotive, } \\
\text { and supportive intervention }\end{array}$ & $\mathrm{SDC}+\mathrm{FLAE}$ & 2.9 & Asian & 6 & 2 \\
\hline 6 & $\begin{array}{l}\text { Protocol for telepsychiatry during } \\
\text { the COVID-19 } \\
\text { pandemic }\end{array}$ & Protocol, psychiatric services & $\mathrm{EC}$ & 11.8 & International & 16 & 6 \\
\hline 7 & $\begin{array}{l}\text { COVID-19 mental health care } \\
\text { toolkit }\end{array}$ & Protocol, clinical assessment & $\mathrm{EC}$ & 8.4 & China & 16 & 2 \\
\hline 8 & $\begin{array}{l}\text { Stigma and discrimination during } \\
\text { the COVID-19 } \\
\text { pandemic* }\end{array}$ & $\begin{array}{l}\text { Viewpoint, stigma and } \\
\text { discrimination, } \\
\text { and underlying factors }\end{array}$ & $\mathrm{SDC}+\mathrm{FLAE}$ & 12.9 & International & 19 & 11 \\
\hline 9 & $\begin{array}{l}\text { A global perspective on } \\
\text { COVID-19 related stigma }\end{array}$ & Viewpoint, global perspective & $\mathrm{EC}$ & 6.8 & India & 16 & 0 \\
\hline 10 & $\begin{array}{l}\text { Alcohol and tobacco use during } \\
\text { COVID-19 } \\
\text { pandemic }\end{array}$ & Review, global perspective & $\mathrm{EC}$ & 3.2 & International & 11 & 0 \\
\hline 11 & $\begin{array}{l}\text { Measuring COVID-19 related } \\
\text { mental health issues: } \\
\text { update* }\end{array}$ & $\begin{array}{l}\text { Review of newly developed } \\
\text { scales: update }\end{array}$ & SDC+FLAE & 3.3 & Australasian & 14 & 0 \\
\hline
\end{tabular}

$S D C$ sequence determines credit, FLAE first and last author emphasis, $E C$ equal credit; research interest: score provided by ResearchGate for individual $\operatorname{article}{ }^{\circledR}$

*Turkey, Pakistan, Germany, Albania (expanded to include)

\#Accessed on March 11, 2021

multiple levels for society's benefit [14]. The COVID-19 pandemic has affected academic activities such as face-to-face conferences, scientific meetings, and grand rounds [15], and published literature has often failed to reach the appropriate people at the appropriate time [16]. However, the extensive use of social media handles, the WPA-ECPs section's social media accounts and newsletter, and the preparation of "Our Science in a Nutshell" series allowed our work to be visible on a larger platform. Still, traditional approaches such as conferences or meetings were also used for dissemination, which took place remotely. As a result, all group members have increased their confidence in sharing relevant knowledge and disseminating their research.

The involvement of countries with different income strata from all WHO regions was very helpful for both the group and each member individually. It allowed gathering, collating, reviewing, and adapting various pieces of information at a truly global level. It also helped to overcome the barriers of publication for LMICs, such as access to publications that would inform discussions, writing in English as a second language, and a lack of research training or a researchoriented environment [17]. It is an advantage of international collaborative research that our team consisted of people with very different language proficiencies, but we complemented each other, and English native speakers and people living in English-speaking countries would proofread the manuscripts.

Communication and discussion have been of fundamental importance. Leads and co-leads always communicated in a timely, regular, and efficient fashion with all team members regarding deadlines, changes, proofreading at every stage of the review, and through the publication process. Similarly, all team members were quick to offer solutions or guidance on drafts, which always led to an improved quality of the draft and speed of its preparation. All discussions were accessible on WhatsApp ${ }^{\circledR}$ and Gmail ${ }^{\circledR}$, giving those who missed a 
discussion an opportunity to review it and provide their contributions when available. This supported ECPs to be engaged with the different stages of manuscript preparation and its publication.

Authorship has been an important part of our publication ethics, and ECPs or early career researchers planning to use our model should be aware of this. Lack of clarity in the authorship sequence or unawareness of the credit system can affect the collaboration's success and lead to discrimination [18]. Two different credit evaluation systems (mixed: sequence determines credit (SDC) + first and last author emphasis (FLAE), equal credit (EC)) were selected by lead and coleads for respective drafts [13]. Though it is often assumed that an experienced person is allocated the last authorship position as a tradition, this can compromise the peer-led models' efficiency. Furthermore, different authorship credit systems exist in different countries [19], and this international variation should be considered. Our experience suggests that adherence to ICMJE criteria and an authorship sequence as per standard credit systems avoids injustice, needless discussion, and discrimination among collaborators.

Even though our model has been quite successful, some challenges need to be acknowledged. For example, on occasions, we could not initiate the clinical research due to a lack of funding, time constraints, and international regulations to share the data. Policymakers, stakeholders, and international organizations (e.g., WPA or WHO) should consider addressing these barriers to accelerate the collaboration and mental health research during a pandemic. Also, most team members were healthcare professionals working clinically at the frontline at different phases of the pandemic, which made for some challenging to contribute to projects. Moreover, due to the multiple responsibilities faced during the pandemic, not all members could lead or co-lead a publication; nonetheless, more than a third of the group has had an opportunity to do it.

Since our work did not entail a specific research project, we have not prepared a formal research protocol in advance. We collected our personal experiences over the last 6 months, but did not use a standardized application form or template to collect these experiences; feedback and suggestions (prepost) were obtained in an informal way. It is difficult to quantify precisely the individual measures' benefits from this model or standardize how each group member has benefited. However, the number of publications from the group and the initiatives for future collaboration can be considered indices of success. It should also be noted that our publications were all related to COVID-19. Most of the COVID-19-related publications were fast-tracked during the review process, although the peer review process varied according to journals processes, which might have impacted the success of the group, being quickly successful with multiple publications. However, it should also be noted that all publications were accepted in peer-reviewed and indexed journals, received with high research interest [20], and many authors have cited them since their publication (Table 2). Furthermore, the success of the small closed group has given rise to another group, referred to as the Global Think Tank. The Global Think Tank has grown to welcome and accept a larger number of participants from all countries. This larger group has developed and published manuscripts on various topics following a similar process to the one here described. This larger number of members also led to the generation of smaller parallel groups working on specific topics initially suggested in the main group.

To conclude, the peer-led model for research and publication proposed and explored in this article can be an effective, easy-to-implement, and cost-effective approach to creating more opportunities for collaborative publications, research, training, and dissemination of results amongst ECPs around the world.

Acknowledgements The authors wish to thank the Early Career Psychiatrists Section of the World Psychiatric Association (WPA) for being a supportive network that allowed to connect early career psychiatrists from different countries to work together on this initiative.

\section{Declarations}

Disclosure On behalf of all authors, the corresponding author states that there is no conflict of interest.

\section{References}

1. Holmes EA, O'Connor RC, Perry VH, Tracey I, Wessely S, Arseneault L, et al. Multidisciplinary research priorities for the COVID-19 pandemic: a call for action for mental health science. Lancet Psychiatry. 2020;7:547-60.

2. Roche GC, Fung P, Ransing R, Noor IM, Shalbafan M, El Hayek S, et al. The state of psychiatric research in the Asia Pacific region. Asia-Pac Psychiatry. 2021;13:e12432.

3. Sharma M, Razzaque B. Research capacity strengthening in South Asia: based on the experience of South Asian Hub for Advocacy, Research and Education on Mental Health (SHARE). Glob Ment Health Camb Engl. 2017;4:e9.

4. Schulze TG. WPA Scientific Sections: a strengthened backbone for the 2020-2023 triennium. World Psychiatry. 2020;19:408-10.

5. Koelkebeck K, Stefanovic MP, Frydecka D, Palumbo C, Andlauer $\mathrm{O}$, Riese $\mathrm{F}$, et al. Barriers and facilitators to conducting research by early career psychiatrists: a literature review. Glob Psychiatry. 2019;2:135-54.

6. Ng RMK, Hermans MHM, Belfort E, Bhugra D. A worldwide survey on training provisions for psychiatric trainees in WPA member associations. Int Rev Psychiatry. 2020;32:98-113.

7. Kram KE, Isabella LA. Mentoring alternatives: the role of peer relationships in career development. Acad Manag J. 1985;28: 110-32. https://doi.org/10.5465/256064.

8. Giancola JK, Heaney MS, Metzger AJ, Whitman B. An organizational-development approach to implementing mentoring partnerships: best practices from physician programs. Consult Psychol J Pract Res. 2016;68:208-21. 
9. Ransing R, Adiukwu F, Pereira-Sanchez V, Ramalho R, Orsolini L, Teixeira ALS, et al. Mental health interventions during the COVID19 pandemic: a conceptual framework by early career psychiatrists. Asian J Psychiatr. 2020;51:102085.

10. Pereira-Sanchez V, Adiukwu F, El Hayek S, Bytyçi DG, GonzalezDiaz JM, Kundadak GK, et al. COVID-19 effect on mental health: patients and workforce. Lancet Psychiatry. 2020;7:e29-30.

11. Ransing R, Ramalho R, Orsolini L, Adiukwu F, Gonzalez-Diaz JM, Larnaout A, et al. Can COVID-19 related mental health issues be measured? Brain Behav Immun. 2020;88:32-4.

12. ICMJE | Recommendations | Defining the Role of Authors and Contributors [Internet]. [cited 2020 Oct 29]. Available from: http://www.icmje.org/recommendations/browse/roles-andresponsibilities/defining-the-role-of-authors-and-contributors.html.

13. Tscharntke T, Hochberg ME, Rand TA, Resh VH, Krauss J. Author sequence and credit for contributions in multiauthored publications. PLoS Biol. 2007;5:e18.

14. Aarons D. Research in epidemic and emergency situations: a model for collaboration and expediting ethics review in two Caribbean countries. Dev World Bioeth. 2018;18:375-84.
15. Kalia V, Srinivasan A, Wilkins L, Luker GD. Adapting scientific conferences to the realities imposed by COVID-19. Radiol Imaging Cancer. 2020;2:e204020.

16. Dong JK, Saunders C, Wachira BW, Thoma B, Chan TM. Social media and the modern scientist: a research primer for low- and middle-income countries. Afr J Emerg Med. 2020;10:S120-124.

17. Salihu Shinkafi T. Challenges experienced by early career researchers in Africa. Future Sci OA Future Sci. 2020;6:FSO469.

18. Desai C. Authorship issues. Indian J Pharm. 2012;44:433.

19. NMC, 2019 [Internet]. 2020 [cited 2020 Oct 29]. Available from: https://www.nmc.org.in/ActivitiWebClient/open/getDocument? path=/Documents/Public/Portal/Gazette/TEQ-17.02.2019.pdf.

20. Research Interest [Internet]. Res. Help Cent. [cited 2021 Jan 31]. Available from: https://explore.researchgate.net/display/support/ Research+Interest.

Publisher's Note Springer Nature remains neutral with regard to jurisdictional claims in published maps and institutional affiliations. 